\title{
Difference in imaging biomarkers between transient and permanent neurological deficits after endovascular treatment of cerebrovascular aneurysms
}

\author{
Yiping Li, MD, ${ }^{1}$ Jason Kim, BS, ${ }^{1}$ Dustin Simpson, MD, ${ }^{1}$ Beverly Aagaard-Kienitz, MD,,2 \\ David Niemann, MD, ${ }^{1}$ Ignatius N. Esene, MD, PhD, MPH, ${ }^{1}$ and Azam Ahmed, MD1 \\ Departments of ${ }^{1}$ Neurological Surgery and ${ }^{2}$ Radiology, University of Wisconsin Medical School, Madison, Wisconsin
}

OBJECTIVE The literature suggests that blood-brain barrier disruption (BBBD) plays a significant role in the development of neurological events in patients with diffusion-weighted imaging (DWI) that is negative for lesions. In this prospective, single-center cohort study, the authors compared the imaging characteristics of patients suffering transient neurological events (TNEs) with those in patients suffering permanent neurological events (PNEs) after having undergone elective embolization of unruptured intracranial aneurysms.

METHODS This prospective cohort study was conducted between July 2016 and June 2019. Inclusion criteria were adults undergoing elective neuroendovascular procedures and the absence of contraindications to MRI. All subjects underwent brain MRI including postcontrast FLAIR (pcFLAIR) sequences for evaluation of BBBD within 24 hours postprocedure.

RESULTS In total, 128 patients harboring 133 unruptured aneurysms were enrolled, 109 of whom (85.2\%) showed some degree of BBBD on pcFLAIR MRI and 50 of whom (39.1\%) suffered an ischemic insult per DWI. In total, 23 patients $(18 \%)$ suffered neurological complications, 16 of which $(12.5 \%)$ were TNEs and 7 of which $(5.5 \%)$ were PNEs. The median extent of BBBD was focal in asymptomatic patients as compared to hemispheric and lobar in the TNE and PNE groups, respectively $(p<0.001)$. The American Society of Anesthesiologists physical status classification predicted the extent of BBBD $(p=0.046)$.

Lesions on DWI were noted in 34 asymptomatic patients (32.4\%) compared to 9 patients (56.3\%) with TNEs and all 7 patients $(100 \%)$ with PNEs $(p<0.001)$. The median number of DWI lesions was 0 (range $0-18$ lesions) in the asymptomatic group compared to 1.5 (range 0-8 lesions) and 8 (range 1-13 lesions) in the TNE and PNE groups, respectively ( $p$ $<0.001)$. Smoking $(p=0.008)$, older age $(p=0.002)$, and longer surgery $(p=0.006)$ were positively associated with the number of lesions on DWI.

On multivariate analysis, intraarterial verapamil $(p=0.02, \mathrm{OR} 8.01,95 \% \mathrm{Cl} 1.35-47.43)$ and extent of BBBD $(p<0.001$, OR $58.58,95 \% \mathrm{Cl} 9.48-361.84$ ) were positively associated with the development of TNEs, while intravenous infusion of midazolam during surgery $(p=0.02$, OR $6.03,95 \% \mathrm{Cl} 1.29-28.20)$ was negatively associated. An increased number of lesions on DWI was the only significant predictor for the development of PNEs $(p<0.001, \mathrm{OR} 49.85,95 \% \mathrm{Cl}$ 5.56-447.10).

CONCLUSIONS An increasing extent of BBBD was associated with the development of TNEs, whereas an increasing number of lesions on DWI was significantly associated with the development of PNEs. BBBD imaging using pcFLAIR may serve as a valuable biomarker for detecting subtle cerebral ischemia and stratifying the risk for ischemic events. https://thejns.org/doi/abs/10.3171/2020.4.JNS192718

KEYWORDS blood-brain barrier disruption; BBB; cerebral aneurysms; endovascular; transient neurological deficits; vascular disorders 
$\mathrm{T}$ HE incidence of blood-brain barrier disruption (BBBD) after elective neuroendovascular procedures is high. ${ }^{1-5}$ Radiographic biomarkers for BBBD include subarachnoid contrast staining on $\mathrm{CT}^{6}$ and leptomeningeal enhancement (LME) on postcontrast FLAIR (pcFLAIR) MRI. ${ }^{7,8}$ Evidence of BBBD on CT has been reported in up to $56 \%$ of patients undergoing elective embolization of intracranial aneurysms (IAs). ${ }^{6} \mathrm{LME}$ on pcFLAIR has been identified in $45 \%$ of patients undergoing mechanical thrombectomy, $57 \%$ of patients after carotid stenting, and up to $83 \%$ of patients undergoing Pipeline embolization of IAs. ${ }^{2-5,9}$ Although BBBD is also found in healthy subjects, numerous case reports and case series have suggested an increasing extent of BBBD as the causative factor in transient neurological events (TNEs) following neuroendovascular surgery. ${ }^{2,10-13}$ A recent prospective cohort study utilizing dynamic contrast-enhanced MRI to identify microvascular permeability in patients presenting with transient ischemic attacks (TIAs) revealed that BBBD was present in $93 \%$ of the cohort including $64.5 \%$ of the patients with negative diffusion-weighted imaging (DWI).${ }^{14}$ Compared to an asymptomatic control group, patients presenting with TIAs were identified as having an increased volume of BBBD, with a greater extent of BBBD also noted in patients with ischemic lesions on DWI. ${ }^{14}$ Altogether, the literature suggests that BBBD plays a significant role in TNEs without DWI lesions as a transient and reversible form of ischemic injury insufficient to modify DWI signal. In this prospective singlecenter study, we compared the imaging characteristics of patients suffering TNEs or permanent neurological events (PNEs) after elective embolization of unruptured IAs and evaluated the influence of BBBD and DWI lesions on clinical outcomes.

\section{Methods \\ Patient Selection}

This prospective cohort study consisted of patients with unruptured IAs undergoing elective endovascular treatment between July 2016 and June 2019. Inclusion criteria were adults undergoing intracranial stent-assisted coil embolization or flow-diversion therapy and the absence of contraindications to MRI. All subjects underwent brain MRI with and without contrast including pcFLAIR sequences for evaluation of BBBD within 24 hours postprocedure. Patient hospital and imaging records were reviewed. This study was approved by the University of Wisconsin Medical School Institutional Review Board. Patient consent was obtained prior to surgical intervention in all cases.

\section{Clinical Data}

Patient demographics, comorbidities, American Society of Anesthesiologists (ASA) physical status classification, procedural details, time between treatment and imaging, and imaging findings were recorded. Neurological deficits identified immediately posttreatment were considered transient if they spontaneously resolved within 72 hours. Deficits persisting for more than 72 hours were categorized as permanent.

\section{Treatment Techniques}

All procedures were performed on a biplane angiographic unit with the patient under general anesthesia (Artis zee, Siemens AX). Systemic blood pressure was monitored continuously and was maintained at clinically desired levels (systolic between 90 and $140 \mathrm{~mm} \mathrm{Hg}$ ).

Contrast was administered for roadmaps, and 2D DSA acquisition was performed using hand injections. 3D DSA acquisitions were obtained using a power injector. Omnipaque 300 (GE Healthcare) was used in all but 1 patient. Verapamil was infused intraarterially for either prevention or treatment of catheter-induced vasospasm.

Endovascular treatment was performed using a transfemoral approach, conventional techniques, and commercially available catheters, balloons, guidewires, stents, and coils. No experimental devices or drugs were used. No patient in this study had an intraprocedural adverse event such as aneurysm rupture, in-stent thrombosis, or parent artery dissection.

\section{Postprocedure Imaging}

The MRI protocol consisted of DWI, noncontrast timeof-flight MRA, pre- and postcontrast T1-weighted, and postcontrast T2-weighted FLAIR (TR $6000 \mathrm{msec}$, TE 135 msec, TI $1700 \mathrm{msec}$, slice thickness $1.6 \mathrm{~mm}$, matrix $256 \times$ 256). Contrast-enhanced images were acquired using 0.1 $\mathrm{mmol} / \mathrm{kg}$ intravenous gadobenate dimeglumine contrast (Multihance, Bracco Diagnostics Inc.).

\section{Imaging Analysis}

All images were stored on and reviewed from our PACS imaging server. PcFLAIR and DWI sequences in axial and coronal planes were independently reviewed by one neuroradiologist (D.S.) and one neurological surgeon (Y.L.). Both reviewers were blinded to patient outcome and treatment techniques.

Extent of BBBD was categorized based on previously published criteria: grade 0 , absent; grade 1 , focal (involving a single or a few sulci but less than half a lobe or cerebellar hemisphere); grade 2, lobar (involving a majority of the frontal, parietal, temporal, or occipital lobe or cerebellar hemisphere); or grade 3, hemispheric (involving more than half of at least 2 of 4 lobes or both cerebellar hemispheres; Fig. 1). ${ }^{2}$ The number and extent of DWI abnormalities were also recorded based on previously published criteria: grade 0 , absent; grade 1, less than 6 foci of restricted diffusion; or grade 2, 6 or more foci of restricted diffusion..$^{23}$ The location of abnormalities was categorized according to conventional arterial territory (right/left anterior cerebral artery, right/left middle cerebral artery, right/left posterior cerebral artery, and right/left cerebellar hemispheres) and compared to the side of aneurysm treatment.

\section{Statistical Analysis}

Summary statistics (such as means and standard deviations) were calculated using Excel data analytics software (Microsoft Corp.). The significance of each variable with the various neurological deficits was assessed using the chi-square test and Fisher's exact test or Mann-Whitney 

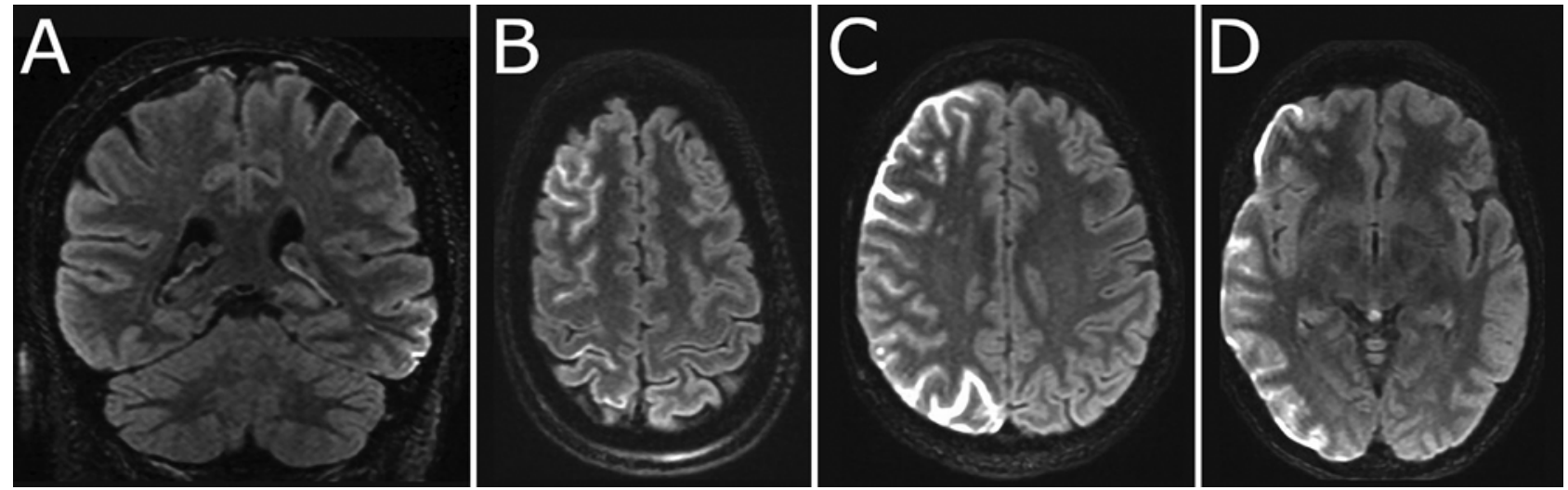

FIG. 1. BBB grading scale. Coronal and axial pcFLAIR MRI demonstrating focal (A), lobar (B), and hemispheric (C and D) extents of BBB impairment.

U-test or Kruskal-Wallis test, as appropriate. To evaluate the relative contributions of clinical and radiographic factors, a receiver operating characteristic curve analysis was run to determine the optimal cutoff value, and then a univariate logistic regression was performed against the presence of each type of neurological deficit. The predictors of the extent of BBBD and number of DWI lesions were determined using univariate and multivariate linear regressions. Significance for variable entry into the multivariate regression was set at 0.10 . A backward stepwise procedure based on likelihood was used to select the variables included in the final model. Odds ratios and 95\% confidence intervals were derived from each variable coefficient in the final model and presented for statistically significant findings when applicable. Statistical analysis was performed using MedCalc (MedCalc Software). A p value $<0.05$ was considered statistically significant.

\section{Results}

\section{Patient Characteristics}

In total, 128 patients harboring 133 unruptured IAs were included in the study. Three patients underwent simultaneous treatment of 2 adjacent aneurysms using a single flow-diverting stent, whereas 2 patients underwent treatment of 2 aneurysms in different arterial territories at different stages. BBBD was assessed during each intervention and independently accounted for when separate staged interventions were performed. The mean patient age was 58.5 years (range $21-84$ years), and $18.8 \%$ of the patients were men. The mean aneurysm diameter was 7.1 $\pm 6.8 \mathrm{~mm}$. No patient had subarachnoid hemorrhage either before or after treatment. Hypertension and smoking were observed in $43.8 \%$ and $39.1 \%$ of patients, respectively. Characteristics such as aneurysm size, contrast dose, BMI, diabetes, hypertension, smoking, renal disease, and coronary artery disease did not differ between the group without complications and those that suffered TNEs or PNEs. The symptomatic groups were more likely to be older ( $p$ $<0.001$ ), to require the use of multiple stents especially flow-diverting stents ( $\mathrm{p}=0.01$ and 0.02 , respectively), and to receive intraarterial verapamil $(\mathrm{p}=0.03)$. Anesthetics and medications used during surgery, including heparin, phenylephrine, ephedrine, rocuronium, sevoflurane, propofol, and fentanyl, did not differ among the groups, whereas the use of midazolam as part of the anesthetic regimen during surgery was more common in the asymptomatic cohort ( $p=0.001)$. Additional patient demographics are shown in Table 1.

\section{Use of Verapamil for Treatment of Catheter-Associated Vasospasm}

In 14 patients, intraarterial verapamil was administered into the parent vessel for the treatment of severe catheterassociated vasospasm. Six of these patients developed postprocedural neurological deficits, while 8 did not $(\mathrm{p}=$ $0.02)$. Five of the 14 patients developed TNEs compared to 9 who did not $(p=0.01)$. Treatment of vasospasm with intraarterial verapamil was not associated with the development of PNEs. The mean dose of intraarterial verapamil for treatment of vasospasm was $12 \pm 3.5 \mathrm{mg}$ (range 8-19 mg).

\section{BBBD and DWI}

Postoperative head CT was performed in 41 cases (32\%), whereas pcFLAIR MRI was obtained in all study subjects. Subarachnoid contrast staining was noted on 12 head CTs (29.3\%), while 109 patients (85.2\%) showed some degree of BBBD on pcFLAIR MRI. In all instances, BBBD was identified ipsilateral to the site of intervention, corresponding to the vascular territory harboring the treated aneurysm. In 11 cases, bilateral disruption was noted consistent with posterior circulation involvement or the presence of robust anterior communicating arteries. Follow-up MRI to evaluate the evolution of the FLAIR signal was available in 42 patients. In 37 of 42 cases $(88.1 \%)$, pcFLAIR signal had resolved over a median follow-up of 3 months (range 2-18 months). Of the 5 cases in which pcFLAIR had not resolved, the signal remained present at 2 weeks in 2 cases and at 3 months in 3 cases. Two patients did not have subsequent follow-up imaging, while the pcFLAIR signal resolved in the other 3 cases over a median follow-up of 6 months (range 6-19 months). On postoperative MRI, 50 patients (39.1\%) had 
TABLE 1. No deficits versus TNEs versus PNEs

\begin{tabular}{|c|c|c|c|c|c|}
\hline & Total & No Deficits & TNEs & PNEs & $p$ Value \\
\hline Mean age in yrs (range) & $58.5(21-84)$ & $57(21-78)$ & $68(40-84)$ & $67(58-79)$ & $<0.001$ \\
\hline Male sex & $24 / 128$ & $22 / 105$ & $1 / 16$ & $1 / 7$ & 0.356 \\
\hline Diabetes & $14 / 128$ & $9 / 105$ & $3 / 16$ & $2 / 7$ & 0.147 \\
\hline Hypertension & $56 / 128$ & $41 / 105$ & $10 / 16$ & $5 / 7$ & 0.067 \\
\hline Median EGFR in $\mathrm{ml} / \mathrm{min} / 1.73 \mathrm{~m}^{2}$ (range) & $88.9(19.6-185.5)$ & $88.9(19.6-185.5)$ & $88.5(27.6-139.6)$ & $92(60-126)$ & 0.863 \\
\hline Median creatinine in mg/dl (range) & $0.7(0.4-2.6)$ & $0.7(0.4-2.6)$ & $0.7(0.5-1.8)$ & $0.7(0.5-1.0)$ & 0.912 \\
\hline Hyponatremia & $0 / 128$ & $0 / 105$ & $0 / 16$ & $0 / 7$ & 0.99 \\
\hline Coronary artery disease & $8 / 128$ & $5 / 105$ & $2 / 16$ & $1 / 7$ & 0.327 \\
\hline Smoking & $50 / 128$ & $46 / 105$ & $3 / 16$ & $1 / 7$ & 0.062 \\
\hline Median largest aneurysm diameter in mm (range) & $5(1-51)$ & $5(1-51)$ & $6(4-25)$ & $4.5(1.7-35)$ & 0.441 \\
\hline Median no. of stents (range) & $0(0-32)$ & $0(0-24)$ & $3(0-7)$ & $2(0-32)$ & 0.010 \\
\hline Flow-diverting stent & $46 / 128$ & $32 / 105$ & $10 / 16$ & $4 / 7$ & 0.022 \\
\hline Vasospasm & $14 / 128$ & $8 / 105$ & $5 / 16$ & $1 / 7$ & 0.018 \\
\hline Median dose of intraarterial verapamil in mg (range) & $3(0-19)$ & $3(0-18)$ & $4(0-19)$ & $3(0-15)$ & 0.027 \\
\hline Visipaque contrast & $2 / 128$ & $2 / 105$ & $0 / 16$ & $0 / 7$ & 0.800 \\
\hline DWI lesions & $50 / 128$ & $34 / 105$ & $9 / 16$ & $7 / 7$ & $<0.001$ \\
\hline Median no. of DWI lesions (range) & $0(0-18)$ & $0(0-18)$ & $1.5(0-8)$ & $8(1-13)$ & $<0.001$ \\
\hline Presence of BBBD & $109 / 128$ & $86 / 105$ & $16 / 16$ & $7 / 7$ & 0.087 \\
\hline Median extent/grade of BBBD (range) & $1.5(0-3)$ & $1(0-3)$ & $3(1-3)$ & $2(1-3)$ & $<0.001$ \\
\hline Contrast staining on CT & $12 / 128$ & $10 / 105$ & $2 / 16$ & $0 / 7$ & 0.634 \\
\hline Median contrast dose in ml (range) & $100(40-310)$ & $90(40-280)$ & $110(50-210)$ & $120(70-310)$ & 0.153 \\
\hline Median length of surgery in mins (range) & $220(71-604)$ & $209(107-542)$ & $235.5(71-300)$ & $266(192-604)$ & 0.078 \\
\hline Median BMI in $\mathrm{kg} / \mathrm{m}^{2}$ (range) & $28(6-46.3)$ & $28(6-46.3)$ & $29.5(21.4-44.2)$ & $25.0(22.8-31)$ & 0.361 \\
\hline Median ASA class (range) & III (I-IV) & III (I-IV) & III (II-IV) & III (II-III) & 0.058 \\
\hline Median heparin dose in units (range) & $8150(0-19,044)$ & $8000(0-19,044)$ & $8150(7000-13,000)$ & $9000(5000-13,000)$ & 0.767 \\
\hline Median phenylephrine dose in $\mu \mathrm{g}$ (range) & $4.4(0-30.4)$ & $4.3(0-30.4)$ & $4.7(0-15.7)$ & $5.1(0.6-11.1)$ & 0.436 \\
\hline Median ephedrine dose in mg (range) & $0(0-60)$ & $0(0-60)$ & $2.5(0-25)$ & $0(0-30)$ & 0.716 \\
\hline Use of rocuronium & $116 / 128$ & $95 / 105$ & $14 / 16$ & $7 / 7$ & 0.634 \\
\hline Use of sevoflurane & $71 / 128$ & $59 / 105$ & $11 / 16$ & $1 / 7$ & 0.051 \\
\hline Use of propofol & $127 / 128$ & $104 / 105$ & $16 / 16$ & $7 / 7$ & 0.895 \\
\hline Use of midazolam & $98 / 128$ & $87 / 105$ & $7 / 16$ & $4 / 7$ & 0.001 \\
\hline Use of fentanyl & $123 / 128$ & $101 / 105$ & $15 / 16$ & $7 / 7$ & 0.771 \\
\hline
\end{tabular}

EGFR = estimated glomerular filtration rate.

Boldface type indicates statistical significance.

suffered an ischemic insult per DWI. The mean number of DWI lesions per patient was $1.6 \pm 3.1$.

The degree of consistency in measuring the BBBD grade between the two raters (D.S., neuroradiology; Y.L., neurointerventional) was expressed using kappa statistics. ${ }^{11}$ The intrarater reliability was $100 \%$, while the chance-adjusted measure of agreement (interrater reliability) was $86.2 \%$ ( $p<0.001$, fixed-marginal kappa statistic). The 9 cases of disagreement were reevaluated by both raters, and the second interrater reliability was $100 \%$.

Testing for an association between the time elapsed from completion of treatment to postoperative MRI and the presence or extent of BBBD revealed no significant difference. In patients without BBBD, the mean time to initiate MRI was $684 \pm 549$ minutes as compared to $519 \pm$ $351,440 \pm 295$, and $719 \pm 593$ minutes for focal, lobar, and hemispheric grades of BBBD, respectively $(\mathrm{p}=0.11)$. The time interval from completion of treatment to MRI was not significantly different among the extents of BBBD.

Regression analyses for variables associated with the extent of BBBD and the number of ischemic lesions on DWI are shown in Tables 2 and 3. In a univariate regression model, increasing age and ASA physical status classification, which is a measure of systemic illness and overall health, were the only significant variables selected for the multivariate stepwise selection method (Table 2). The final multivariate model identified ASA class as the only significant association with the extent of BBBD $(p=0.46)$. Interestingly, the mean number of DWI lesions per patient overall was $1.6 \pm 3.1$, compared to $0.9 \pm 1.6$ per patient for focal, $0.8 \pm 1.7$ for lobar, and $3.3 \pm 3.3$ for hemispheric grades of BBBD; however, there was no statistically sig- 
TABLE 2. Predictors of the presence and/or extent of BBBD

\begin{tabular}{|c|c|c|c|c|c|c|}
\hline \multirow[b]{2}{*}{ Variable } & \multicolumn{3}{|c|}{ Univariate Analysis } & \multicolumn{3}{|c|}{ Multivariate Analysis } \\
\hline & Coefficient & SE & $p$ Value & Coefficient & SE & $p$ Value \\
\hline Age & 0.012 & 0.006 & 0.048 & 0.009 & 0.006 & 0.12 \\
\hline Hypertension & - & - & NS & & & \\
\hline Smoking & - & - & NS & & & \\
\hline No. of stents & - & - & NS & & & \\
\hline Vasospasm & - & - & NS & & & \\
\hline Intraarterial verapamil & - & - & NS & & & \\
\hline Length of surgery & - & - & NS & & & \\
\hline DWI lesions & - & - & NS & & & \\
\hline No. of DWI lesions & - & - & NS & & & \\
\hline ASA class & 0.274 & 0.136 & 0.046 & 0.274 & 0.136 & 0.046 \\
\hline Flow-diverting stent & - & - & NS & & & \\
\hline Use of sevoflurane & - & - & NS & & & \\
\hline Use of midazolam & - & - & NS & & & \\
\hline
\end{tabular}

NS = not significant; $\mathrm{SE}=$ standard error.

Boldface type indicates statistical significance.

nificant association between the number of DWI lesions and extent of BBBD $(\mathrm{p}=0.22)$.

With respect to regression analysis for variables associated with the number of ischemic lesions on DWI (Table 3 ), several variables were selected for the final multivariate model using a backward stepwise process. Smoking $(p=0.008)$, older age $(p=0.002)$, and increased length of surgery $(p=0.006)$ were positively associated with the presence and/or number of lesions on DWI. The presence and extent of BBBD were not significantly associated with the presence or extent of lesions on DWI.

\section{Neurological Outcomes}

A total of 23 patients (18\%) suffered neurological com- plications, 16 of which (12.5\%) were TNEs and 7 of which (5.5\%) were PNEs. The cutoff between TNE and PNE was a duration of 72 hours. The severity of TNEs ranged from global aphasia, hemianopia, and confusion to hemiparesis, aphasia, and gaze deviation. The mean National Institutes of Health Stroke Scale (NIHSS) score for patients with TNE was 9.1 \pm 5.3 (range 4-21), and the mean duration of TNEs was $37.6 \pm 15.8$ hours (range 12-66 hours). The severity of PNEs ranged from dysarthria, facial droop, and arm weakness to hemiplegia, delirium, and neglect. The mean NIHSS score for patients with PNEs was $5.9 \pm 3.3$ (range 4-14), and the mean duration of PNEs was $33.3 \pm$ 40.1 days (range 4 days-permanent).

In all patients with TNEs and PNEs, the presence of

TABLE 3. Predictors of the presence and/or number of DWI lesions

\begin{tabular}{|c|c|c|c|c|c|c|}
\hline \multirow[b]{2}{*}{ Variable } & \multicolumn{3}{|c|}{ Univariate Analysis } & \multicolumn{3}{|c|}{ Multivariate Analysis } \\
\hline & Coefficient & SE & $\mathrm{p}$ Value & Coefficient & SE & $p$ Value \\
\hline Age & 0.067 & 0.020 & $<0.001$ & 0.058 & 0.019 & 0.002 \\
\hline Hypertension & - & - & NS & & & \\
\hline Smoking & 1.512 & 0.542 & 0.006 & 1.369 & 0.509 & 0.008 \\
\hline No. of stents & 0.182 & 0.057 & 0.002 & - & - & NS \\
\hline Vasospasm & - & - & NS & & & \\
\hline Intraarterial verapamil & 0.141 & 0.070 & 0.045 & - & - & NS \\
\hline Length of surgery & 0.010 & 0.003 & 0.003 & $<0.001$ & 0.003 & 0.006 \\
\hline BBBD & - & - & NS & & & \\
\hline Extent of BBBD & - & - & NS & & & \\
\hline ASA class & - & - & NS & & & \\
\hline Flow-diverting stent & - & - & NS & & & \\
\hline Use of sevoflurane & - & - & NS & & & \\
\hline Use of midazolam & -1.434 & 0.630 & 0.024 & - & - & NS \\
\hline
\end{tabular}

Boldface type indicates statistical significance. 


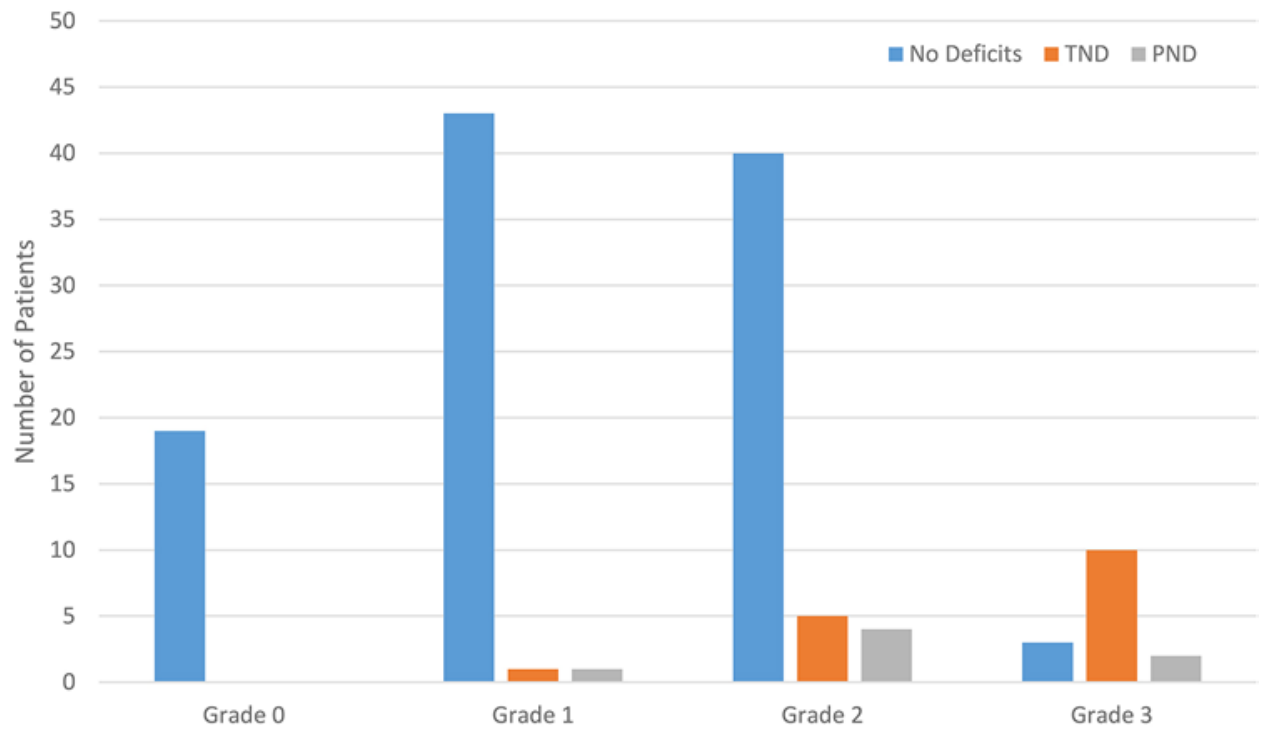

FIG. 2. Bar graph highlighting the association between the extent of BBBD and development of neurological deficits. PND = permanent neurological deficit; TND = transient neurological deficit. Figure is available in color online only.

BBBD was noted (Fig. 2). The median extent of BBBD was focal in asymptomatic patients compared to hemispheric and lobar in the TNE and PNE groups, respectively ( $\mathrm{p}<$ $0.001)$. Lesions on DWI were noted in 34 asymptomatic patients $(32.4 \%)$ compared to 9 patients $(56.3 \%)$ with TNEs and all 7 patients $(100 \%)$ with PNEs ( $\mathrm{p}<0.001)$. Of the 9 patients with TNEs, lesions on DWI were identified in the precentral gyrus in 2, visual cortex in 2, and deep white matter in 3 and involving noneloquent locations (insula and anterior frontal lobe) in 2 patients. The median number of DWI lesions was 0 (range 0-18 lesions) in the asymptomatic group compared to 1.5 (range 0-8 lesions) and 8 (range 1-13 lesions) in the TNE and PNE groups, respectively $(\mathrm{p}<0.001$; Table 1$)$.

Regression analysis for variables associated with TNEs and PNEs are shown in Tables 4 and 5. With respect to TNEs, several variables were selected for the final multivariate model using a backward stepwise process. The final multivariate model showed intraarterial verapamil $(\mathrm{p}=$ 0.02, OR 8.01, 95\% CI 1.35-47.43) and extent of BBBD (p $<0.001$, OR 58.58, 95\% CI 9.48-361.84) were positively associated, while intravenous infusion of midazolam during surgery ( $\mathrm{p}=0.02$, OR $6.03,95 \%$ CI $1.29-28.20$ ) was negatively associated with the development of TNEs (Table 4).

With respect to regression analysis for variables associated with the development of PNEs, univariate analysis identified increasing age and number of DWI lesions as the only significant variables selected for the multivariate stepwise selection method (Table 5). The final multivariate model identified an increased number of DWI lesions as the only significant association with the development of PNEs ( $p<0.001$, OR 49.85, 95\% CI 5.56-447.10).

\section{Discussion}

In this study, we used an MRI protocol to study the relationship between BBBD and neurological complications in patients undergoing endovascular treatment of unruptured IAs. To the best of our knowledge, this is the first report comparing the extent of BBBD and ischemia between TNEs and PNEs after endovascular surgery. Consistent with the literature, we report a high proportion of both BBBD and ischemic lesions on DWI in symptomatic and asymptomatic patients undergoing endovascular surgery. ${ }^{1,3-5,9,14,16-19}$ We found that a greater extent of BBBD was associated with the development of TNEs, whereas an increased number of lesions on DWI was significantly associated with the development of PNEs. Higher doses of intraarterial verapamil and lower doses of intravenous midazolam given during surgery were also associated with greater odds of developing TNEs. While there was no statistically significant association between the number of DWI lesions and extent of BBBD, in the final multivariate model, ASA grade was significantly associated with the extent of BBBD. Smoking, older age, and increased length of surgery were positively associated with the presence and/or greater number of lesions on DWI, which is consistent with previously established risk factors.

The association between BBBD and TNEs has many important clinical implications. Most TNEs after endovascular surgery are traditionally thought to be ischemic in nature resulting in temporary but reversible cerebral dysfunction in the form of TIAs. DWI remains the goldstandard technique for detecting microischemic lesions of the brain; ${ }^{36}$ however, DWI alone is often unreliable, as the frequency of DWI positivity in TIA ranges between $9 \%$ and $67 \%{ }^{20-22}$ The sensitivity and specificity of DWI in detecting permanent stroke is reported to be $90 \%-98 \%$ and $100 \%$, respectively. ${ }^{2,23}$ In our cohort, lesions on DWI were detected in only $56.3 \%$ of patients with TNEs, which is consistent with the previous literature on TIAs. In contrast, in our study $100 \%$ of the patients suffering from PNEs had lesions on DWI. On multivariate regression analysis, the 
TABLE 4. Receiver operating characteristic and regression analysis of predictors of TNEs

\begin{tabular}{|c|c|c|c|c|c|c|c|c|c|}
\hline \multirow[b]{2}{*}{ Variable } & \multicolumn{3}{|c|}{ ROC Analysis } & \multicolumn{3}{|c|}{ Univariate Logistics } & \multicolumn{3}{|c|}{ Multivariate Logistics } \\
\hline & ROC AUC & Associated Criterion & p Value & OR & $95 \% \mathrm{Cl}$ & p Value & OR & $95 \% \mathrm{Cl}$ & $p$ Value \\
\hline Age & 0.68 & $>66$ & 0.020 & 5.6 & $1.9-16.7$ & 0.002 & - & - & NS \\
\hline Hypertension & - & - & - & - & - & NS & & & \\
\hline Smoking & - & - & - & - & - & NS & & & \\
\hline Race & - & - & - & - & - & NS & & & \\
\hline No. of stents & 0.67 & $>2$ & 0.020 & 5.3 & $1.8-15.7$ & 0.003 & - & - & NS \\
\hline Vasospasm & - & - & - & 5.2 & $1.5-18.3$ & 0.01 & - & - & NS \\
\hline Intraarterial verapamil & 0.69 & $>3$ & 0.007 & 5.8 & $1.5-21.4$ & 0.009 & 8.0 & $1.35-47.4$ & 0.022 \\
\hline DWI lesions & & & & - & - & NS & & & \\
\hline No. of DWI lesions & - & - & NS & & & & & & \\
\hline BBBD & - & - & - & - & - & NS & & & \\
\hline Extent of BBBD & 0.87 & $>2$ & $<0.001$ & 35.7 & $9.2-137.9$ & $<0.001$ & 58.6 & $9.5-361.8$ & $<0.001$ \\
\hline ASA class & 0.65 & $>2$ & 0.029 & 0.3 & $0.1-1.0$ & 0.048 & - & - & NS \\
\hline Flow-diverting stent & - & - & - & 3.5 & $1.2-10.4$ & 0.023 & - & - & NS \\
\hline Use of sevoflurane & - & - & - & - & - & NS & & & \\
\hline Use of midazolam & - & - & - & 5.6 & $1.9-16.7$ & 0.002 & 6.0 & $1.3-28.2$ & 0.022 \\
\hline
\end{tabular}

$\mathrm{AUC}=$ area under the curve; $\mathrm{ROC}=$ receiver operating characteristic.

Boldface type indicates statistical significance.

TABLE 5. Receiver operating characteristic and regression analysis of predictors of PNEs

\begin{tabular}{|c|c|c|c|c|c|c|c|c|c|}
\hline \multirow[b]{2}{*}{ Variable } & \multicolumn{3}{|c|}{ ROC Analysis } & \multicolumn{3}{|c|}{ Univariate Logistics } & \multicolumn{3}{|c|}{ Multivariate Logistics } \\
\hline & ROC AUC & Associated Criterion & $p$ Value & OR & $95 \% \mathrm{Cl}$ & $p$ Value & OR & $95 \% \mathrm{Cl}$ & $\mathrm{p}$ Value \\
\hline Age & 0.82 & $>63$ & $<0.001$ & 14.7 & $1.7-127.0$ & 0.014 & - & - & NS \\
\hline Hypertension & - & - & NS & & & & & & \\
\hline Smoking & - & - & - & - & - & NS & & & \\
\hline Race & - & - & - & - & - & NS & & & \\
\hline No. of stents & - & - & - & - & - & NS & & & \\
\hline Vasospasm & - & - & - & - & - & NS & & & \\
\hline Intraarterial verapamil & - & - & NS & & & & & & \\
\hline DWI lesions & - & - & - & - & - & NS & & & \\
\hline No. of DWI lesions & 0.93 & $>3$ & $<0.001$ & 49.8 & $5.5-447.1$ & $<0.001$ & 49.85 & $5.56-447.1$ & $<0.001$ \\
\hline BBBD & - & - & - & - & - & NS & & & \\
\hline Extent of BBBD & 0.72 & $>1$ & 0.008 & - & - & NS & & & \\
\hline ASA class & - & - & NS & & & & & & \\
\hline Flow-diverting stent & - & - & - & - & - & NS & & & \\
\hline Use of sevoflurane & - & - & - & - & - & NS & & & \\
\hline Use of midazolam & - & - & - & - & - & NS & & & \\
\hline
\end{tabular}


presence and/or number of lesions on DWI was predictive of PNEs, whereas the extent of BBBD was predictive of TNEs. The association between BBBD and TNEs suggests that BBBD could represent a transient and reversible form of ischemic injury insufficient to modify DWI signal.

BBBD is widely recognized as a prominent feature of cerebral ischemia and has been investigated as a therapeutic target. ${ }^{24}$ If subtle transient ischemia is the underlying mechanism of TNEs, then our findings suggest that endothelial cells may be more prone to ischemic injury than parenchymal cells. Contrary to DWI, which often indicates irreversible ischemic injury, BBBD may be a biomarker for a reversible ischemic process to help guide patient management and counseling. In the present series, all patients with PNEs had lesions on DWI. In contrast, 7 of the 16 patients (44\%) with TNEs were void of DWI lesions. Of these 7 patients, all had a lobar or hemispheric extent of BBBD (4 hemispheric and 3 lobar). In addition, 31 of 49 patients (63.3\%) with lobar and 6 of 15 patients $(40 \%)$ with hemispheric BBBD did not have lesions on DWI. Therefore, BBBD imaging using pcFLAIR may be more sensitive in detecting subtle and transient ischemic lesions of the brain than DWI and serve as a valuable biomarker in risk stratification for ischemic events..$^{14}$ Identifying patients with BBBD may also have a potential role in distinguishing those at risk during endovascular surgery and those likely to suffer recurrent stroke when presenting with a TIA..$^{14}$

Previous authors have elucidated the role of BBBD in cerebral ischemia using dynamic contrast and pcFLAIR imaging and have found worse clinical outcomes in those with extensive BBBD. ${ }^{8,14,22}$ Serlin et al. conducted a prospective cohort study evaluating the extent of BBBD in patients presenting with TIA in comparison to that in healthy controls. ${ }^{14}$ Using dynamic contrast MRI, these authors found a significantly higher volume of BBBD in the patients with TIA than in healthy controls including 64.5\% of patients with negative DWI. The authors concluded that the BBBD-involved brain areas correspond more with clinical presentation than with DWI, that therefore BBBD likely reflects an underlying microvascular pathology, and that BBBD could signify a reversible ischemic injury that underlies TNEs. ${ }^{14}$ Also consistent with our findings, Suthiphosuwan et al. assessed the relationship between various clinical factors and BBBD after elective endovascular surgery and found no association between BBBD and the presence of DWI lesions, volume of iodinated contrast, or timing of MRI relative to the conclusion of surgery. ${ }^{4}$ These authors similarly identified BBBD only in the vascular distribution consistent with the corresponding site of intervention; contrary to our findings, however, they found increased BBBD with longer procedure times and more angiographic runs. In addition, we report the association of systemic illness (ASA class) with the development of BBBD consistent with previously established risk factors. ${ }^{11,17,25,26}$ Our findings that smoking, older age, and longer surgery were positively associated with the development of lesions on DWI is also consistent with thromboembolic risk factors of endovascular surgery and the development of stroke..$^{27-29}$

Dose-dependent intraarterial administration of verapamil, a calcium channel blocker and vasodilator that is commonly used in endovascular surgery to prevent or treat catheter-induced cerebral vasospasm, was also identified as an independent predictor for developing TNEs. Verapamil was used to treat catheter-associated vasospasm in 14 patients, all of whom developed BBBD and 6 of whom developed neurological deficits. There was a significantly higher proportion of TNEs in patients treated for vasospasm than in those not treated for vasospasm, but identification and treatment of vasospasm was not significantly associated with the development of TNEs on multivariate regression. These findings suggest that verapamil administration alone is unlikely to be the underlying cause of BBBD but rather a surrogate for compromises in intraprocedural blood flow. Mechanistically, transient episodes of ischemia may induce BBBD and the subsequent development of TNEs. Territorial ischemia can be the result of luminal obstruction due to the use of large-bore guide catheters especially during catheterization of smaller vertebral or distal cerebral arteries. The occurrence of unrecognized or untreated mild to moderate vasospasm or the use of prophylactic verapamil in preventing catheter-associated vasospasm in cases of tortuous anatomy or during catheterization of smaller-caliber parent arteries may also result in higher rates of transient flow limitation and subsequent ischemic and thromboembolic complications, accounting for the higher rates of TNEs observed in association with verapamil administration. Maneuvers aimed at preventing cerebral vasospasm during catheter navigation or the use of smaller-bore guide catheters may be prudent in preventing temporary luminal encumbrances..$^{17,26}$

Of the anesthetics evaluated, a significant inverse relationship was identified between the administration of midazolam and the development of TNEs. Indications for midazolam use in neuroanesthesia include premedication for the treatment of anxiety or as an induction agent given its minimal effects on the cardiovascular and respiratory system. The administration of midazolam at our institution is left to the discretion of the anesthetist; therefore, selection bias cannot be ruled out as an influence on the observed reduction in TNEs. The underlying etiology of the observed association between lower rates of TNEs and midazolam administration is unknown. Sedatives and anesthetics such as propofol and opiates are associated with differential emergence from anesthesia. ${ }^{15,30}$ The focal neurological deficits seen during differential awakening can often persist for hours after emergence from anesthesia and can be amplified in the setting of underlying transient ischemic injury. We hypothesize that the use of midazolam as an alternate induction agent may reduce the need for other sedatives or anesthetics with higher rates of differential emergence thereby confounding the perceived rate of TNEs. ${ }^{30}$ It is also conceivable that midazolam has neuroprotective effects during episodes of transient ischemia, as it has been implicated in suppressing excitatory amino acids triggered by cerebral ischemia and produces dose-related reductions in cerebral blood flow demand and oxygen consumption; however, further studies are needed to validate this association. ${ }^{31-35}$

\section{Study Limitations}

This was a cohort study and is thus limited by the lack 
of a control group. It was a single-center study and may be biased by regional variations in patient populations, anesthetic use, and neuroendovascular techniques. TNEs are often underreported; therefore, the results may be subject to reporting bias. An inherent feature of studies on diagnostic performance is observer bias resulting in interrater disagreement. This was evaluated in our study by restricting the MRI rating to two independent investigators. The interobserver reliability of detecting and grading BBBD was excellent enough $(\mathrm{kappa}=86.2 \%)$ for our results to be considered reproducible.

\section{Conclusions}

An increased extent of BBBD was associated with the development of TNEs, whereas a greater number of lesions on DWI was significantly associated with the development of PNEs, suggesting that imaging biomarkers for detecting BBBD may be more sensitive in detecting subtle and transient ischemic lesions of the brain that may not be potent enough to alter DWI signal. BBBD imaging using pcFLAIR may serve as a valuable biomarker in detecting subtle cerebral ischemia and stratifying risk for ischemic events.

\section{Acknowledgments}

We would like to sincerely thank Charles Strother, MD, for comments that greatly improved the manuscript. Professional statistical consultation was obtained from the University of Wisconsin Biostatistics Department with sponsorship from the Cancer Center Support Grant.

\section{References}

1. Enomoto Y, Yoshimura S, Yamada K, Iwama T. Convulsion during intra-arterial infusion of fasudil hydrochloride for the treatment of cerebral vasospasm following subarachnoid hemorrhage. Neurol Med Chir (Tokyo). 2010;50(1):7-12.

2. Li Y, Funk C, Dawkins D, et al. Leptomeningeal enhancement is associated with transient neurologic deficits after flow diversion of intracranial aneurysms. World Neurosurg. 2018;120:e94-e99.

3. Renú A, Laredo C, Lopez-Rueda A, et al. Vessel wall enhancement and blood-cerebrospinal fluid barrier disruption after mechanical thrombectomy in acute ischemic stroke. Stroke. 2017;48(3):651-657.

4. Suthiphosuwan S, Hsu CC, Bharatha A. HARMless: Transient cortical and sulcal hyperintensity on gadoliniumenhanced FLAIR after elective endovascular coiling of intracranial aneurysms. AJNR Am J Neuroradiol. 2018;39(4):720726.

5. Warach S, Latour LL. Evidence of reperfusion injury, exacerbated by thrombolytic therapy, in human focal brain ischemia using a novel imaging marker of early blood-brain barrier disruption. Stroke. 2004;35(11)(suppl 1):2659-2661.

6. Ozturk A, Saatci I, Pamuk AG, et al. Focal increased cortical density in immediate postembolization CT scans of patients with intracranial aneurysms. AJNR Am J Neuroradiol. 2006;27(9):1866-1875.

7. Landis JR, Koch GG. The measurement of observer agreement for categorical data. Biometrics. 1977;33(1):159-174.

8. Lee KM, Kim JH, Kim E, et al. Early stage of hyperintense acute reperfusion marker on contrast-enhanced FLAIR images in patients with acute stroke. AJR Am J Roentgenol. 2016;206(6):1272-1275.
9. Ogami R, Nakahara T, Hamasaki O, et al. Cerebrospinal fluid enhancement on fluid attenuated inversion recovery images after carotid artery stenting with neuroprotective balloon occlusions: hemodynamic instability and blood-brain barrier disruption. Cardiovasc Intervent Radiol. 2011;34(5):936-941.

10. Gold JP, Charlson ME, Williams-Russo P, et al. Improvement of outcomes after coronary artery bypass. A randomized trial comparing intraoperative high versus low mean arterial pressure. J Thorac Cardiovasc Surg. 1995;110(5):1302-1314.

11. Hussain M, Berger M, Eckenhoff RG, Seitz DP. General anesthetic and the risk of dementia in elderly patients: current insights. Clin Interv Aging. 2014;9:1619-1628.

12. Iosif C, Lecomte JC, Pedrolo-Silveira E, et al. Evaluation of ischemic lesion prevalence after endovascular treatment of intracranial aneurysms, as documented by 3-T diffusionweighted imaging: a 2 -year, single-center cohort study. $J$ Neurosurg. 2018;128(4):982-991.

13. Kuroiwa T, Ting P, Martinez H, Klatzo I. The biphasic opening of the blood-brain barrier to proteins following temporary middle cerebral artery occlusion. Acta Neuropathol. 1985;68(2):122-129.

14. Serlin Y, Ofer J, Ben-Arie G, et al. Blood-brain barrier leakage: a new biomarker in transient ischemic attacks. Stroke. 2019;50(5):1266-1269.

15. Lin N, Han R, Zhou J, Gelb AW. Mild sedation exacerbates or unmasks focal neurologic dysfunction in neurosurgical patients with supratentorial brain mass lesions in a drugspecific manner. Anesthesiology. 2016;124(3):598-607.

16. Klötzsch C, Nahser HC, Henkes H, et al. Detection of microemboli distal to cerebral aneurysms before and after therapeutic embolization. AJNR Am J Neuroradiol. 1998;19(7):1315-1318

17. Elkassabany NM, Bhatia J, Deogaonkar A, et al. Perioperative complications of blood brain barrier disruption under general anesthesia: a retrospective review. J Neurosurg Anesthesiol. 2008;20(1):45-48.

18. Freeman LR, Keller JN. Oxidative stress and cerebral endothelial cells: regulation of the blood-brain-barrier and antioxidant based interventions. Biochim Biophys Acta. 2012;1822(5):822-829.

19. Wolf O, Heider P, Heinz M, et al. Microembolic signals detected by transcranial Doppler sonography during carotid endarterectomy and correlation with serial diffusion-weighted imaging. Stroke. 2004;35(11):e373-e375.

20. Brazzelli M, Chappell FM, Miranda H, et al. Diffusionweighted imaging and diagnosis of transient ischemic attack. Ann Neurol. 2014;75(1):67-76.

21. Lipton SA, Rosenberg PA. Excitatory amino acids as a final common pathway for neurologic disorders. $N$ Engl J Med. 1994;330(9):613-622.

22. Nadarajan V, Perry RJ, Johnson J, Werring DJ. Transient ischaemic attacks: mimics and chameleons. Pract Neurol. 2014;14(1):23-31.

23. Kang DH, Kim BM, Kim DJ, et al. MR-DWI-positive lesions and symptomatic ischemic complications after coiling of unruptured intracranial aneurysms. Stroke. 2013;44(3):789-791.

24. Villringer K, Sanz Cuesta BE, Ostwaldt AC, et al. DCE-MRI blood-brain barrier assessment in acute ischemic stroke. Neurology. 2017;88(5):433-440.

25. Montagne A, Barnes SR, Sweeney MD, et al. Blood-brain barrier breakdown in the aging human hippocampus. Neuron. 2015;85(2):296-302.

26. Zlokovic BV. Neurodegeneration and the neurovascular unit. Nat Med. 2010;16(12):1370-1371.

27. Albayram S, Selcuk H, Kara B, et al. Thromboembolic events associated with balloon-assisted coil embolization: evaluation with diffusion-weighted MR imaging. AJNR Am J Neuroradiol. 2004;25(10):1768-1777.

28. Goldstein LB, Bushnell CD, Adams RJ, et al. Guidelines for 
the primary prevention of stroke: a guideline for healthcare professionals from the American Heart Association/American Stroke Association. Stroke. 2011;42(2):517-584.

29. Shah RS, Cole JW. Smoking and stroke: the more you smoke the more you stroke. Expert Rev Cardiovasc Ther. 2010;8(7):917-932.

30. Dubow J, Bernstein RA. Amplification of acute focal ischemic deficit by narcotics. Neurocrit Care. 2008;8(3):427-429.

31. Ito H, Watanabe $\mathrm{Y}$, Isshiki A, Uchino H. Neuroprotective properties of propofol and midazolam, but not pentobarbital, on neuronal damage induced by forebrain ischemia, based on the GABAA receptors. Acta Anaesthesiol Scand. 1999;43(2):153-162.

32. Kahn RA, Panah M, Weinberger J. Modulation of ischemic excitatory neurotransmitter and gamma-aminobutyric acid release during global temporary cerebral ischemia by selective neuronal nitric oxide synthase inhibition. Anesth Analg. 1997;84(5):997-1003.

33. Liu L, You Q, Tu Y, et al. Midazolam inhibits the apoptosis of astrocytes induced by oxygen glucose deprivation via targeting JAK2-STAT3 signaling pathway. Cell Physiol Biochem. 2015;35(1):126-136.

34. Schulte am Esch J, Kochs E. Midazolam and flumazenil in neuroanaesthesia. Acta Anaesthesiol Scand Suppl. 1990;92:96-102.

35. Yamada K, Goto S, Yoshikawa M, Ushio Y. Gabaergic transmission and tyrosine hydroxylase expression in the nigral dopaminergic neurons: an in vivo study using a reversible ischemia model of rats. Neuroscience. 1996;73(3):783-789.

36. van Everdingen KJ, van der Grond J, Kappelle LJ, et al. Diffusion-weighted magnetic resonance imaging in acute stroke. Stroke. 1998;29(9):1783-1790.

\section{Disclosures}

The authors report no conflict of interest concerning the materials or methods used in this study or the findings specified in this paper.

\section{Author Contributions}

Conception and design: Li. Acquisition of data: Li, Kim, Simpson. Analysis and interpretation of data: Li, Kim, Simpson, Esene, Ahmed. Drafting the article: Li, Kim, Esene, Ahmed. Critically revising the article: Li, Aagaard-Kienitz, Niemann, Ahmed. Reviewed submitted version of manuscript: Li, Kim, Simpson, Aagaard-Kienitz, Niemann, Ahmed. Approved the final version of the manuscript on behalf of all authors: Li. Statistical analysis: Kim, Ahmed. Administrative/technical/material support: Ahmed. Study supervision: Ahmed.

\section{Supplemental Information}

Current Affiliations

Dr. Esene: Neurosurgery Division, Faculty of Health Sciences, University of Bamenda, Republic of Cameroon.

\section{Correspondence}

Yiping Li: University of Wisconsin-Madison, WI. liyip218@ gmail.com. 